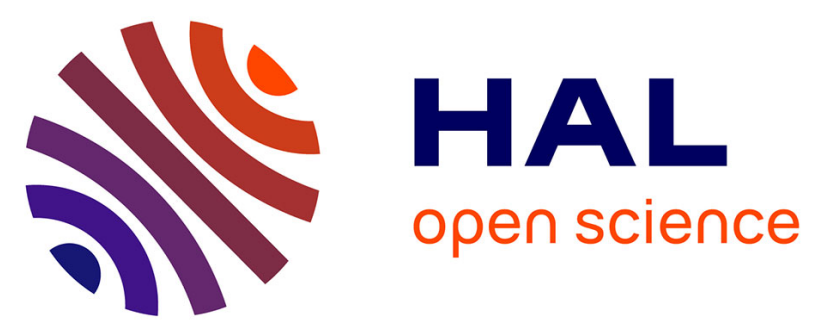

\title{
Injection of Calcium Phosphate Apatitic Cement/Blood Composites in Intervertebral Fusion Cages
}

\author{
Henrique Gonçalves, Hugues Pascal-Moussellard, Julie Lesoeur, Verena \\ Schnitzler, Borhane H. Fellah, Nastassia M.S. M S Wagner, Charlotte Mellier, \\ Jean-Michel Bouler, Bruno Bujoli, Olivier Gauthier
}

\section{- To cite this version:}

Henrique Gonçalves, Hugues Pascal-Moussellard, Julie Lesoeur, Verena Schnitzler, Borhane H. Fellah, et al.. Injection of Calcium Phosphate Apatitic Cement/Blood Composites in Intervertebral Fusion Cages: a simple and efficient alternative to autograft leading to enhanced spine fusion. Spine, 2020, 45 (20), pp.E1288-E1295. 10.1097/BRS.0000000000003598 . hal-02994427

\section{HAL Id: hal-02994427 https://hal.science/hal-02994427}

Submitted on 7 Nov 2020

HAL is a multi-disciplinary open access archive for the deposit and dissemination of scientific research documents, whether they are published or not. The documents may come from teaching and research institutions in France or abroad, or from public or private research centers.
L'archive ouverte pluridisciplinaire HAL, est destinée au dépôt et à la diffusion de documents scientifiques de niveau recherche, publiés ou non, émanant des établissements d'enseignement et de recherche français ou étrangers, des laboratoires publics ou privés. 


\section{Injection of calcium phosphate apatitic cement /blood composites in intervertebral fusion cages: a simple and efficient alternative to autograft leading to enhanced spine fusion.}

H. Gonçalves, ${ }^{\text {a }}$ H. Pascal-Moussellard, ${ }^{b}$ J. Lesoeur, ${ }^{c}$ V. Schnitzler, ${ }^{\text {d B. H. Fellah, }}{ }^{\text {a N.M.S. }}$ Wagner, ${ }^{a}$ C. Mellier, ${ }^{\mathrm{d}} \mathrm{J}-\mathrm{M}$. Bouler, ${ }^{\mathrm{e}}$ B. Bujoli*e and O. Gauthier*a,c

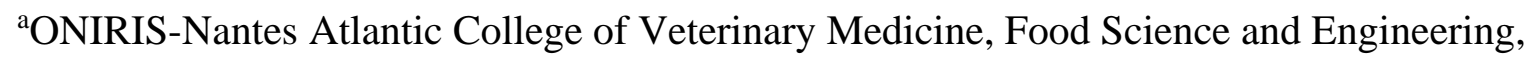
Nantes, France, ${ }^{\mathrm{b}}$ Service d'orthopédie, Hôpital de La Pitié-Salpêtrière, 47-83, Boulevard de 1'Hôpital, 75013 Paris, France, ${ }^{\text {'INSERM UMR 1229, University of Nantes, France, }{ }^{\text {d} G r a f t y s ~}}$ SA, Eiffel Park, Bâtiment D, 415 Rue Claude Nicolas Ledoux, Pôle d'activités d'Aix en Provence, 13854 Aix en Provence CEDEX 3, France, ${ }^{e}$ Université de Nantes, CNRS, CEISAM, UMR 6230, F-44000 Nantes, France

Address correspondence and reprint requests to Bruno BUJOLI, Université de Nantes, CEISAM, UMR CNRS 6230, 2 Rue de la Houssinière, BP92208, 44322 Nantes Cedex 03, France. Email: bruno.bujoli@univ-nantes.fr

\section{Study design}

An animal model of anterior lumbar arthrodesis was designed to investigate whether a calcium phosphate cement (CPC) mixed with autologous whole blood might favourably compete with autograft for enhanced spine fusion

\section{Objective}


The objective of this study was to use a large animal model to investigate a new approach for spine fusion which consists in the injection of a highly resorbable CPC / blood composite to fill the interbody fusion cage, once placed in the intervertebral space.

\section{Summary of background data}

Spinal fusion via intervertebral arthrodesis is a common surgical procedure for which the gold standard consists in filling the internal hollow part of the cage with bone autograft. However, this approach suffers from some drawbacks, including a second surgical site which requires additional time spent in the surgical room, and the occurrence of additional new pain on the bone harvesting site along with risks of long-term morbidity.

\section{Methods}

An animal model of anterior lumbar arthrodesis involved 14 sheep that received a fusion cage in two non-adjacent lumbar levels. For one-half of the animals, the cages were filled after impaction by injection of a CPC/blood composite in one level and the blood-free CPC reference in the other. For the other seven animals, the CPC/blood composite was compared to bone autograft.

\section{Results}

SEM and histological examination of the explants gave evidence of a good osteointegration for the three types of implanted materials. For the first group, no spine fusion was observed for the blood-free CPC reference while a highly successful spine fusion (6/7 cages) was present for the CPC/blood composite, three months after surgery. For the second group, successful arthrodesis was observed for $57 \%$ (4/7) of cages filled with iliac autograft, while the fusion rate was $71 \%$ (5/7) in the case of cages filled with the CPC/blood composite.

\section{Conclusion}


This study reports that the observed fusion rate when filling the cage with a calcium phosphate cement mixed with autologous whole blood was at least as good as results obtained with autograft. Once placed in the intervertebral space, the cage was easily and homogeneously filled with the $\mathrm{CPC} /$ blood composite, and this new strategy might thus offer a less invasive option for intervertebral spine fusion

\section{Level of evidence : N/A}

\section{Introduction}

Low back pain has an incidence rate of about 50 to $80 \%$ of the global population and a high socioeconomic impact, since it is the first cause of disability before age $45[1,2]$. In case of failure of conservative treatments, lumbar arthrodesis is one common surgical approach[3, 4], including intervertebral arthrodesis for which an interbody fusion cage is inserted between two adjacent vertebrae[5], after discectomy. The gold standard consists in filling the internal hollow part of the cage with autologous bone graft, to favor and promote spine fusion. However, the use of autograft suffers from some drawbacks[6-8], including (i) a second surgical site which requires additional time spent in the surgical room, (ii) the occurrence of additional new pain on the bone harvesting site and risks of long-term morbidity, (iii) the usual loss of autograft during impaction of the pre-filled cage in the intervertebral space, which may hamper the bone fusion due to a limited contact between the two vertebral plateaus and the bone graft.

In this paper, an alternative has been investigated which consists in the injection of a synthetic self-setting bone substitute to fill the interbody fusion cage, once placed in the intervertebral space. Provided that performances at least as good as autologous bone graft are obtained[9, 10], this approach might offer: $(i)$ a less invasive and safer surgical protocol, since no autograft is needed, (ii) a complete filling of the hollow part of the cage with the biomaterial which should 
favor an optimal contact between the bone substitute and the two vertebral plateaus. We have first designed a reliable and reproducible animal model of anterior lumbar arthrodesis, suitable to compare the performances of the bone substitutes selected for this study versus a bone autograft. Sheep was chosen since it offers advantages including its size, spinal anatomy and biomechanics rather close to the human situation[11-14], as well as an easy management at a reasonable cost.

We recently reported promising results using an injectable calcium phosphate cement (CPC) / blood composite, which showed very quick resorption and remodeling of the material by bone cells, when implanted in bone critical-sized defects [15]. In the present study, 14 animals were implanted at two non-adjacent levels with human cervical PEEK cages that had the suitable dimensions for our animal model. The ability of the CPC / blood composite to promote spine fusion was first compared to a blood-free CPC reference, three months after implantation, using half of the animals. Then, the remaining animals were used to compare the performances of the CPC / blood composite with autologous bone graft.

\section{Materials and methods}

\subsection{Investigated bone substitutes}

The Graftys ${ }^{\circledR}$ Quickset reference is used in clinics and was obtained from Graftys SA. The CPC/blood composite investigated in this study is an experimental material. The composition and preparation of the two materials are described in the Supplementary data.

\subsection{Design of the animal model}

14 adult female Vendeen sheep with comparable size (average body weight: $60 \mathrm{~kg}$ ) were used in this study. Polyetheretherketone (PEEK) non-resorbable and radiolucent cages (ROI-C ${ }^{\circledR}$ from LDR Medical, Troyes, France) for human cervical arthrodesis by an anterior approach were selected, since they had the appropriate dimensions for the developed animal model $(14 \times$ 
$14 \mathrm{~mm}, 5$ or $6 \mathrm{~mm}$ in height). Animal handling and surgical procedures are described in the Supplementary data. Seven animals were implanted to compare the CPC/autologous blood composite versus the blood-free CPC. Given that the biological activity of the CPC/blood composite was previously shown to express after a short term period when implanted in bone critical-sized defects,[15] a short implantation time (3 months) was selected to verify that the same discrimination between the CPC/blood composite and the Graftys ${ }^{\circledR}$ Quickset reference was also present in this model. Results were compared using $\mu$-CT and SEM measurements, and histological analyses. Then the comparison between the CPC/blood composite and the autologous bone graft was performed using the other seven animals, that were kept six months postoperatively, since this is the shortest implantation time usually mentioned in the literature to obtain bone fusion. The long-term effects and fusion rate obtained with the two biomaterials was compared using qualitative SEM and histological analyses. To minimize the possible effect of inter-animal variation, two different implantation sites per animal were used, and each animal received two different biomaterials, with a randomization of the biomaterial allocation. This also allowed to reduce the number of animals for ethical reasons to comply with the $3 \mathrm{R}$ rule (Reduce, Refine, Replace), while ensuring at the same time statistical analysis of the results and preserving the scientific relevance of the study.

\subsection{Data acquisition and analyses}

A full-blind ex vivo analysis of the samples was performed by the same team for both groups. Details about $\mu$-CT, SEM and histological analyses are given in the Supplementary data

\section{Results}

Fourteen ewes were operated on two non-adjacent lumbar levels (usually $\mathrm{L}_{2}-\mathrm{L}_{3}$ and $\mathrm{L}_{4}-\mathrm{L}_{5}$ ). On one-half of the animals the superior and lower levels received an empty cage in which injection of the CPC/blood composite was performed in one level and the blood-free CPC reference in 
the other (Figure 1), and the ability of the two compositions to promote spine fusion was compared three months after implantation. On the other seven animals, the respective performances of the CPC / blood composite and the autograft were investigated six months after implantation.

For the two groups, no complication occurred during the postoperative period. Animals were sacrificed and after explantation of the bone segments, manual evaluation did not reveal any macroscopic intervertebral mobility at the operated levels while the adjacent levels were still mobile. All the implanted cages were still in place, with no expulsion. SEM evaluation of the explants was performed on sagittal sections centered on the radiolucent cage and allowed to visualize the cage walls (in dark) in the intervertebral space and assess continuity of bone and therefore fusion between the two adjacent vertebral levels. The results are summarized in Table 1, showing the absence of any spine fusion within the cages filled with the blood-free CPC reference (0/7). By contrast, successful spine fusion was most often present for cages filled with either autograft (4/7) or the CPC/blood composite (11/14).

Figure 2 illustrates two examples of successful fusion: one with a cage filled with the autograft (Figure 2a), the other filled by injection of the CPC/blood composite (Figures $2 \mathrm{~b}$ and $2 \mathrm{c}$ ). By contrast, Figure 3 shows two samples for which no spine fusion occurred: one with a cage filled with the autograft (Figure 3a), the other filled by injection of the CPC reference (Figure 3b).

Results obtained for the blood-free CPC versus the CPC/blood composite were compared using 3D $\mu$-CT tomodensitometric analysis three months after surgery (Figure 4). New bone formation into the cage represented $21.85 \pm 10.41 \%$ of the cage volume for the blood-free cement versus $41.81 \pm 4.13 \%$ for the $\mathrm{CPC} /$ blood composite $(\mathrm{p}=0.016)$. This was consistent with the remaining amount of cement which represented $33.29 \pm 1.36 \%$ of the cage volume for the blood-free CPC versus $10.05 \pm 4.18 \%$ for the $\mathrm{CPC} /$ blood composite $(\mathrm{p}=0.031)$. Interestingly, the cement resorption and the new bone formation into the cages showed a 
significant correlation, with $r=0.72\left(\mathrm{IC}_{95 \%}\right.$ [0.309;09054]; $\left.\mathrm{p}=0.0036\right)$, regardless of the cement used (Figure S1 in Supporting data). These quantitative 3D $\mu$-CT measurements were not significantly different from the 2D ones (data not shown).

Quantitative 2D SEM results provided similar results than $\mu$-CT. New bone formation into the cage represented $18.43 \pm 2.48 \%$ of the cage volume for the blood-free CPC versus $35.28 \pm$ $12.28 \%$ for the $\mathrm{CPC} / \mathrm{blood}$ composite $(\mathrm{p}=0.004)$. A significantly more important cement resorption was confirmed for the $\mathrm{CPC} / \mathrm{blood}$ composite compared to the blood-free $\mathrm{CPC}$, since the remaining amount of cement represented $31.07 \pm 3.08 \%$ of the cage volume for the bloodfree $\mathrm{CPC}$ versus $8.53 \pm 11.50 \%$ for the $\mathrm{CPC} /$ blood composite $(\mathrm{p}=0.001)$. In conclusion, the results obtained from 2D quantitative SEM analysis and from 3D $\mu$-CT measurements were consistent in terms of amount of newly-formed bone and percentage of cement degradation, whatever the considered cement.

HE staining was first performed, for which bone and bone-free areas are colored in blue-violet and pale pink, respectively. Figure 5 illustrates results obtained for cages filled using (i) autologous bone graft, for which interbody fusion was successful (Figure 5a) or failed (Figure $5 b$ ), as evidenced for the latter case by the presence of two pale pink non mineralized edgings on both sides of the implant. The bone graft seemed however to be viable, although no bone continuity was present; (ii) the blood-free CPC which did not provide any bone fusion although new bone formation was visible into the cage (Figure 5c); (iii) the CPC/blood composite which led to bone fusion with a new trabecular bone network bridging one vertebral body to the other, with however small residual cement fragments surrounded by newly formed (Figure 5d).

Movat's pentachrome staining allowed to assess the mineralization and maturity of the newlyformed bone. Figure 6a shows the example of successful bone fusion with a cage filled with autologous bone graft, for which a bone continuity between adjacent vertebral bodies can be observed thanks to the formation of a well-mineralized trabecular bone network. Figure 6c 
corresponds to the same observation made for a cage filled with the $\mathrm{CPC} / \mathrm{blood}$ composite, except that small areas of residual cement can be observed, surrounded by well-mineralized newly formed bone (Figure 7). By contrast, Figure $6 \mathrm{~b}$ gives evidence of the situation for a cage filled with the blood-free CPC, showing a fragmentation of the cement with visible bone ingrowth coming from the vertebral bodies, although no fusion occurred.

\section{Discussion}

The efficacy of injectable calcium phosphate cement-based biomaterials has been investigated as interbody fusion cages fillers for intervertebral lumbar arthrodesis, by comparison with the autograft gold standard. Sheep lumbar spine was selected for this preclinical investigation, since it has been shown to be a suitable model for human spinal research[16], with recent reports in the literature.[17-20]

In this work, we have developed an original retroperitoneal anterior approach for intervertebral lumbar arthrodesis[21] and PEEK radiolucent cages for human cervical arthrodesis were found to be well adapted to the morphology of sheep lumbar vertebral body.

The commercial Graftys ${ }^{\circledR}$ Quickset [abbreviated as QS] which is used in clinics and has proven efficiency in traumatology[22-24], was found to be ineffective three months after surgery. Although part of the CPC was degraded with new bone ingrowth, no spine fusion was observed (0/7 cages), due to the slow degradation rate of the CPC. This was in sharp contrast with the results obtained for the $\mathrm{CPC} / \mathrm{blood}$ composite for which spine fusion was highly successful (6/7 cages), and SEM and histological examination of the explants showed that most of the composite has been degraded and replaced by mature new bone. Moreover, the cement degradation was positively correlated to the new bone formation within the inserted cages. No radio-transparent separation could be seen at the bone/CPC interface, giving evidence of a good osteointegration[22]. Light microscopy showed the absence of any inflammatory infiltrate confirming the cyto- and histocompatibility of both cements. Qualitatively, viable osteoblasts 
were visible on the surface of the new bone trabeculae (Figure 7) in both cases, even though the blood-free CPC showed far more residual cement fragments (Figure 6b).

The reason why the particular composition of CPC/blood composite investigated in this study showed a significantly higher resorption rate than the QS reference was recently reported.[15] The higher particle size of the powder component present in the $\mathrm{CPC} / \mathrm{blood}$ composite $(12 \mathrm{~mm}$ versus $5 \mathrm{~mm}$ for QS) together with the proteins contained in blood both slowed down the CDA nucleation process which is the driving force of the cement setting reaction. As a consequence, the setting reaction only started after 15 hours (compared to 5 minutes for QS), so that the formation of a blood clot took place in the intergranular space prior to the precipitation of apatite. The $\alpha$-TCP particles were thus embedded in the 3D blood clot and this confinement led to a slower crystal growth and the formation of an inorganic network of low density made of very large apatite crystals. In addition, upon swelling when forming, the blood clot led to a ten times larger separation between adjacent calcium phosphate particles in the cement paste. Hence, this particular microstructure favoured colonization by bone cells which then resulted in quicker resorption and remodeling of the material. It is important to note that QS combined to blood exhibited the same resorption rate than the QS reference, and the addition of blood in that case did not modify the setting time nor the microstructure.[15] This means that although the proteins and growth factors present in blood might contribute to promote the in vivo degradation of the cement, the major reason for the quicker resoption of the CPC/blood composite was its microstructure.

Given these promising results, it was relevant to compare the biological behavior of the $\mathrm{CPC} / \mathrm{blood}$ composite with that of autograft which is the gold standard used in clinics for spine fusion. Successful arthrodesis was observed for 57\% (4/7) of cages filled with iliac autograft, which is consistent with results from the literature reporting a $20-58 \%$ fusion rate for anterior lumbar arthrodesis in sheep, less than the success rate in humans[11, 19]. In the case of cages 
filled with the $\mathrm{CPC} /$ blood composite, the observed fusion rate was $71 \%(5 / 7)$, showing performances at least as good as autograft, that might offer a less invasive and safer surgical protocole, since no autograft is needed. However, in the case of the CPC/blood composite, a significant amount of remaining CPC particles were still present, even though the fusion had occurred. The short implantation time investigated here is therefore a limitation of our study and a longer implantation time would be needed to compare the long-term stability of the fusion rate obtained with the autologous bone graft and our CPC-blood approach.

\section{Conclusion}

An alternative to autograft for intervertebral arthrodesis has been investigated which consisted in the impaction of an empty interbody fusion cage which was subsequently filled by injection of a calcium phosphate cement combined to blood, leading to an optimal contact between the bone substitute and the two vertebral plateaus. The observed fusion rate was found to be at least as good as for the control experiment made using pre-filled cages with autograft. Therefore this strategy might offer a less invasive option for intervertebral spine fusion, allowing a reduction of surgical time, the prevention of morbidity effects due to the bone graft harvesting procedure, and the minimization of the risk of graft loosening and degradation related to the impaction of the pre-filled cage. Moreover, even though BMPs have been reported to be efficient in promoting bone fusion similarly to autologous bone graft, they also led to massive ectopic bone formation particularly in spine surgery[25-28] that questioned the interest and safety of BMPs for such an indication. Consequently, as autologous bone graft is still the gold standard material to promote bone fusion, our CPC-blood approach might be an alternative while avoiding the potential adverse effects of BMPs. To confirm the potential of this original strategy, clinical trials would now be needed. It is worth noting that given some predicate CPC/blood composites (e.g. Zimmer Accufill BSM) are already proposed to surgeons, a 510K procedure might be 
implemented for bone void filling applications and, if successful, followed by a specific IDE in intervertebral cages applications in order to confirm the interest of this approach in a safe way.

\section{Disclosure of conflicts of interests}

Some authors of this publication have research support from Graftys SA, approved by both CNRS and the University of Nantes in accordance with their policy on objectivity in research.

\section{Disclosure of Off-Label Use/Unapproved Drugs or Products}

The FDA approved Graftys ${ }^{\circledR}$ Quickset used in this study, is however not approved for intervertebral arthrodesis. The CPC/blood composite is an investigational product with no approval.

Acknowledgements. This work was partially supported by OSEO (FUI 13 - grant F1210010 - SpineInject project), and the Graftys company.

\section{Key Points}

-An alternative to autograft for intervertebral arthrodesis based on a calcium phosphate cement (CPC) combined to blood has been established using an original intervertebral lumbar arthrodesis in a sheep model.

-Thanks to the injectability of the CPC / blood composite, a complete and homogeneous filling of the intervertebral fusion cage was easily achieved after placing the cage in the intervertebral space.

-The plasticity of the $\mathrm{CPC} /$ blood composite led to an optimal contact between the bone substitute and the two vertebral plateaus. 
-The fusion rate obtained for the CPC/blood composite was at least as good as results obtained with autograft.

\section{References}

[1] J. Gourmelen, J.F. Chastang, A. Ozguler, J.L. Lanoe, J.F. Ravaud, A. Leclerc, Frequency of low back pain among men and women aged 30 to 64 years in France. Results of two national surveys, Annales de readaptation et de medecine physique : revue scientifique de la Societe francaise de reeducation fonctionnelle de readaptation et de medecine physique, 50 (2007) 640-644, 633-649.

[2] G. Waddell, Low back disability. A syndrome of Western civilization, Neurosurgery clinics of North America, 2 (1991) 719-738.

[3] F.B. Christensen, Lumbar spinal fusion. Outcome in relation to surgical methods, choice of implant and postoperative rehabilitation, Acta orthopaedica Scandinavica. Supplementum, 75 (2004) 2-43.

[4] V. Goz, J.H. Weinreb, F. Schwab, V. Lafage, T.J. Errico, Comparison of complications, costs, and length of stay of three different lumbar interbody fusion techniques: an analysis of the Nationwide Inpatient Sample database, Spine Journal, 14 (2014) 2019-2027.

[5] T.A. Zdeblick, F.M. Phillips, Interbody cage devices, Spine, 28 (2003) S2-S7.

[6] G.M. Calori, M. Colombo, E.L. Mazza, S. Mazzola, E. Malagoli, G.V. Mineo, Incidence of donor site morbidity following harvesting from iliac crest or RIA graft, Injury-International Journal of the Care of the Injured, 45 (2014) S116-S120. 
[7] R. Dimitriou, G.I. Mataliotakis, A.G. Angoules, N.K. Kanakaris, P.V. Giannoudis, Complications following autologous bone graft harvesting from the iliac crest and using the RIA: A systematic review, Injury-International Journal of the Care of the Injured, 42 (2011) S3-S15.

[8] J.A. Goulet, L.E. Senunas, G.L. DeSilva, M. Greenfield, Autogenous iliac crest bone graft Complications and functional assessment, Clinical Orthopaedics and Related Research, (1997) 76-81.

[9] E. Garcia-Gareta, M.J. Coathup, G.W. Blunn, Osteoinduction of bone grafting materials for bone repair and regeneration, Bone, 81 (2015) 112-121.

[10] P.V. Giannoudis, H. Dinopoulos, E. Tsiridis, Bone substitutes: An update, Injury-International Journal of the Care of the Injured, 36 (2005) 20-27.

[11] I.H. Drespe, G.K. Polzhofer, A.S. Turner, J.N. Grauer, Animal models for spinal fusion, The spine journal : official journal of the North American Spine Society, 5 (2005) 209S-216S.

[12] A. Kettler, L. Liakos, B. Haegele, H.J. Wilke, Are the spines of calf, pig and sheep suitable models for pre-clinical implant tests?, European Spine Journal, 16 (2007) 2186-2192.

[13] S.-R. Sheng, X.-Y. Wang, H.-Z. Xu, G.-Q. Zhu, Y.-F. Zhou, Anatomy of large animal spines and its comparison to the human spine: a systematic review, European Spine Journal, 19 (2010) 46-56.

[14] H.J. Wilke, A. Kettler, L.E. Claes, Are sheep spines a valid biomechanical model for human spines?, Spine, 22 (1997) 2365-2374.

[15] C. Mellier, F.-X. Lefevre, F. Fayon, V. Montouillout, C. Despas, M. Le Ferrec, F. Boukhechba, A. Walcarius, P. Janvier, M. Dutilleul, O. Gauthier, J.-M. Bouler, B. Bujoli, A straightforward approach to enhance the textural, mechanical and biological properties of injectable calcium phosphate apatitic cements (CPCs): CPC/blood composites, a comprehensive study, Acta Biomaterialia, 62 (2017) 328339.

[16] M. Mageed, D. Berner, H. Julke, C. Hohaus, W. Brehm, K. Gerlach, Is sheep lumbar spine a suitable alternative model for human spinal researches? Morphometrical comparison study, Laboratory animal research, 29 (2013) 183-189. 
[17] Y. Li, Z.-g. Wu, X.-k. Li, Z. Guo, S.-h. Wu, Y.-q. Zhang, L. Shi, S.-h. Teoh, Y.-c. Liu, Z.-y. Zhang, A polycaprolactone-tricalcium phosphate composite scaffold as an autograft-free spinal fusion cage in a sheep model, Biomaterials, 35 (2014) 5647-5659.

[18] B.S. Shamsul, K.K. Tan, H.C. Chen, B.S. Aminuddin, B.H.I. Ruszymah, Posterolateral spinal fusion with ostegenesis induced BMSC seeded TCP/HA in a sheep model, Tissue \& Cell, 46 (2014) 152-158.

[19] L.A. Solchaga, C.K. Hee, D.J. Aguiar, J. Ratliff, A.S. Turner, H.B. Seim, III, J.O. Hollinger, L.B. Snel, S.E. Lynch, Augment Bone Graft Products Compare Favorably With Autologous Bone Graft in an Ovine Model of Lumbar Interbody Spine Fusion, Spine, 37 (2012) E461-E467.

[20] D.L. Wheeler, D.C. Fredericks, R.F. Dryer, H.W. Bae, Allogeneic mesenchymal precursor cells (MPCs) combined with an osteoconductive scaffold to promote lumbar interbody spine fusion in an ovine model, Spine Journal, 16 (2016) 389-399.

[21] K.C. McGilvray, E.I. Waldorff, J. Easley, H.B. Seim, N. Zhang, R.J. Linovitz, J.T. Ryaby, C.M. Puttlitz, Evaluation of a polyetheretherketone (PEEK) titanium composite interbody spacer in an ovine lumbar interbody fusion model: biomechanical, microcomputed tomographic, and histologic analyses, Spine J., 17 (2017) 1907-1916.

[22] M. Ollivier, A.M. Gay, A. Cerlier, A. Lunebourg, J.N. Argenson, S. Parratte, Can we achieve bone healing using the diamond concept without bone grafting for recalcitrant tibial nonunions?, Injury, 46 (2015) 1383-1388.

[23] M. Ollivier, M. Turati, M. Munier, A. Lunebourg, J.-N. Argenson, S. Parratte, Balloon tibioplasty for reduction of depressed tibial plateau fractures: Preliminary radiographic and clinical results, International Orthopaedics, 40 (2016) 1961-1966.

[24] A.A. Young, L. Neyton, D.C. Molony, P. Boileau, G. Walch, Glenoid tricortical iliac crest structural bone graft enhanced with resorbable cement for the treatment ofaseptic glenoid loosening, Techniques in Shoulder \& Elbow Surgery, 12 (2011) 12-17.

[25] N.E. Epstein, Complications due to the use of BMP/INFUSE in spine surgery: The evidence continues to mount, Surgical neurology international, 4 (2013) S343-352. 
[26] A. Faundez, C. Tournier, M. Garcia, S. Aunoble, J.-C. Le Huec, Bone morphogenetic protein use in spine surgery-complications and outcomes: a systematic review, International Orthopaedics, 40 (2016) 1309-1319.

[27] T.R. Khan, K.R. Pearce, S.J. McAnany, C.M. Peters, M.C. Gupta, L.P. Zebala, Comparison of transforaminal lumbar interbody fusion outcomes in patients receiving rhBMP-2 versus autograft, Spine Journal, 18 (2018) 439-446.

[28] C.A. Tannoury, H.S. An, Complications with the use of bone morphogenetic protein 2 (BMP-2) in spine surgery, Spine Journal, 14 (2014) 552-559.

Figure 1. Main surgical steps in the sheep model of anterior lumbar arthrodesis. (a) Discectomy (bolded arrow). (b) PEEK intervertebral cage on its insertion device and with a predrilled hole (*) to allow cement injection. (c) Position of the intervertebral cage in the intervertebral space, with perioperative injection of the cement into thecage through a 18-gauge needle . (d) Perioperative view of the CPC/blood composite injection.
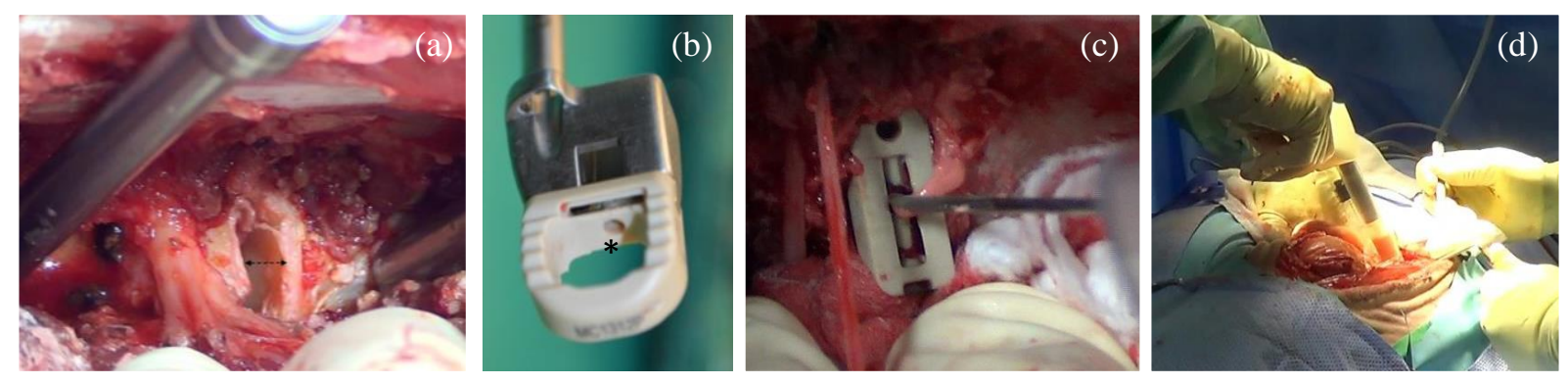

Figure 2. SEM images of successful bone fusions 6 months after implantation: (a) cage inserted between $\mathrm{L}_{4}$ and $\mathrm{L}_{5}$ vertebral bodies and filled with autologous bone graft, showing in addition 
a ventral bone fusion between the two vertebral bodies; (b) cage inserted between $\mathrm{L}_{2}$ and $\mathrm{L}_{3}$ vertebral bodies filled with the CPC/autologous blood composite. Note the new trabecular network developed into the cage bridging one vertebral body to the other; only a few CPC particles (red square) are visible (original magnification $\times 20$ ); (c) higher magnification of the selected area (red square) showing the presence of residual CPC particles (*)
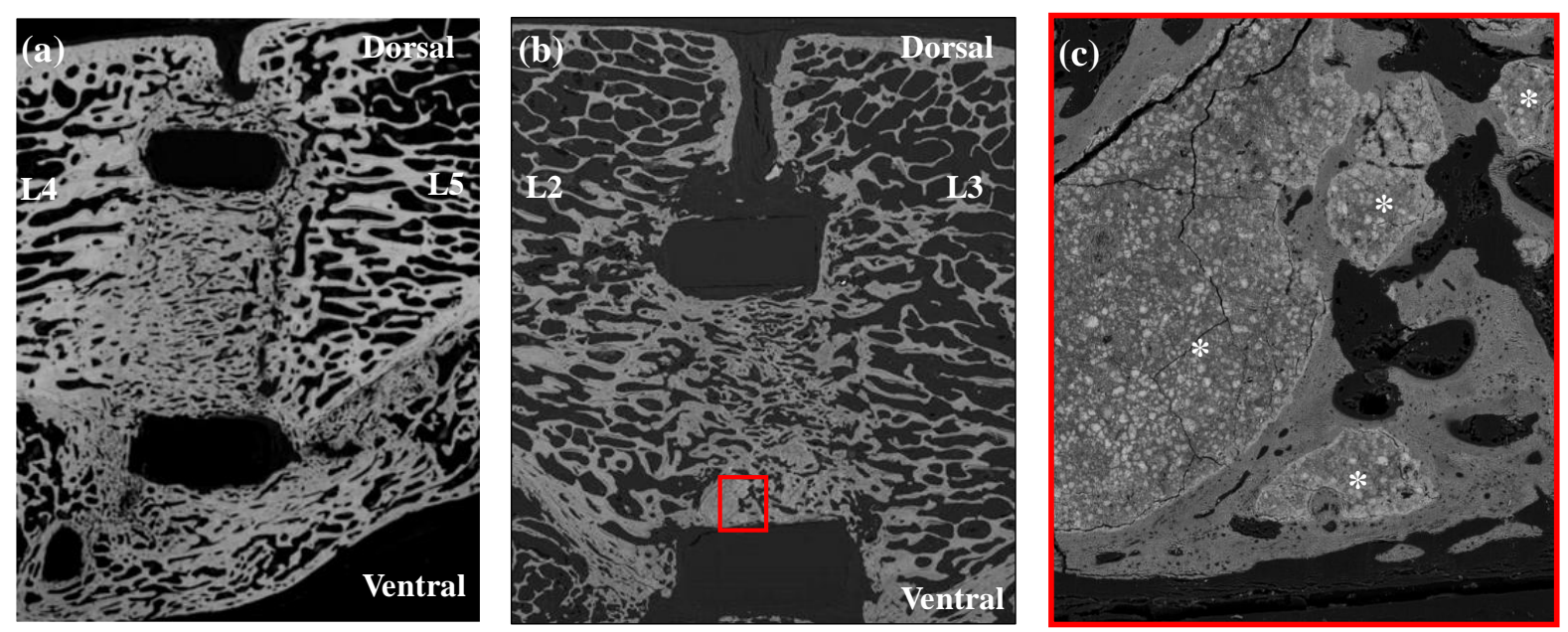

Figure 3. SEM images of failed bone fusions: (a) cage inserted between $\mathrm{L}_{4}$ and $\mathrm{L}_{5}$ vertebral bodies and filled with autologous bone graft for which a clear discontinuity is present between the two vertebral segments, 6 months after implantation,; (b) cage inserted between $\mathrm{L}_{4}$ and $\mathrm{L}_{5}$ vertebral bodies and filled with the blood-free CPC for which a high amount of residual CPC fragments can be observed, 3 months after implantation. Note the cement fragments which are still visible with a satisfactory peripheral osteoconduction and bone ingrowth $(\rightarrow)$, but new bone formation did not occur in the center of the intervertebral space $(*)$. Both cases showed evidence of non-union. Original magnification $\times 20$. 

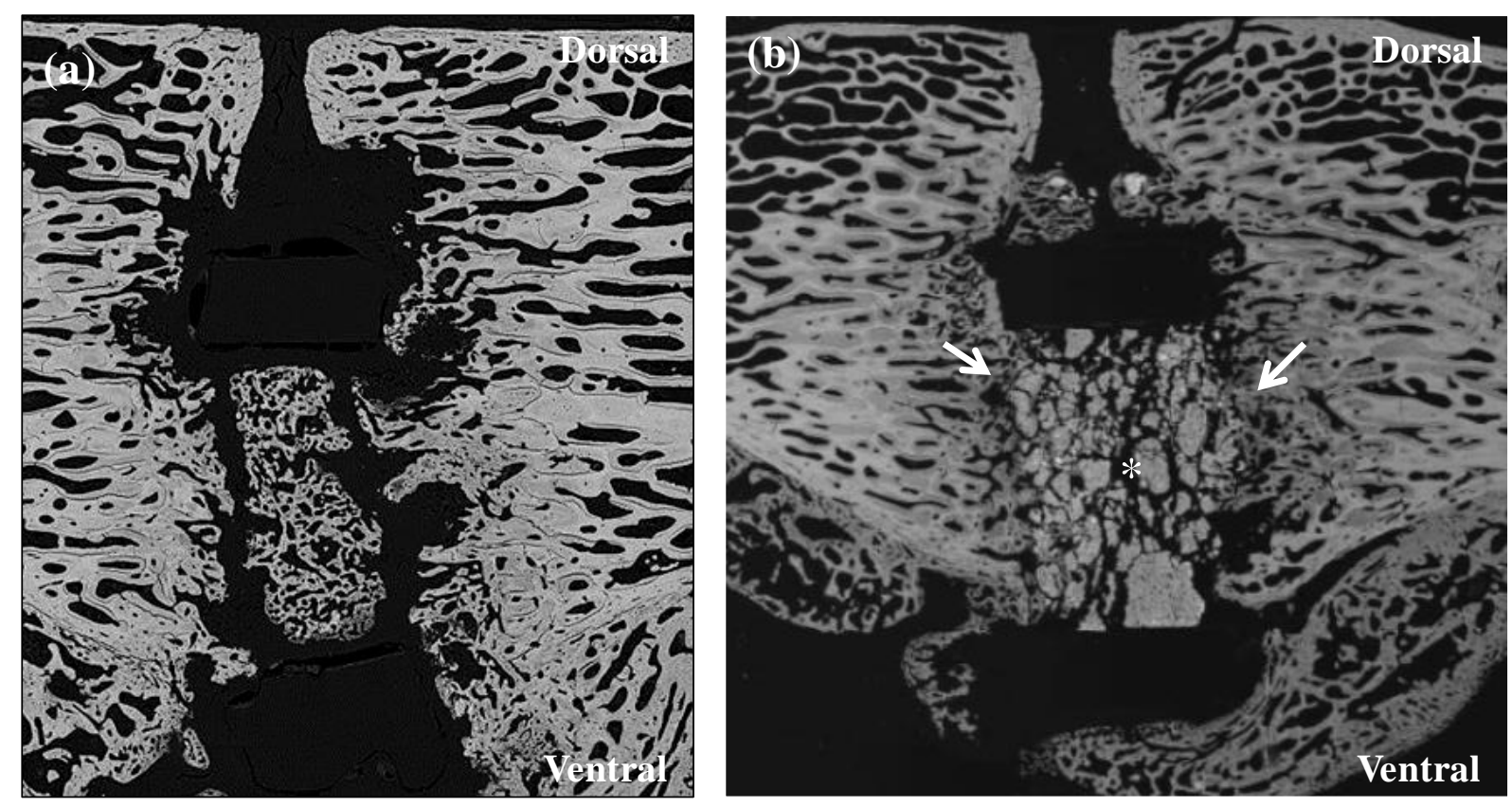

Figure 4. $\mu-\mathrm{CT}$ analysis: (a) Lateral image showing the position of the cage in the $\mathrm{L}_{2}-\mathrm{L}_{3}$ intervertebral space. Note the metal tag included in the lateral wall of the PEEK cage, and the dorsal and ventral cage walls figured by the two arrowheads; (b) 2D $\mu$-CT transverse image of a cage positioned into the $\mathrm{L}_{2}-\mathrm{L}_{3}$ intervertebral space and filled with the CPC-blood composite. The radiolucent rectangular frame of the cage appears clearly. Only a few particles of cement remain visible (light grey *), and most of the cage cavity appears filled with newly-formed bone with a contrast similar to the adjacent vertebral bone. Original magnification $\times 20$. 

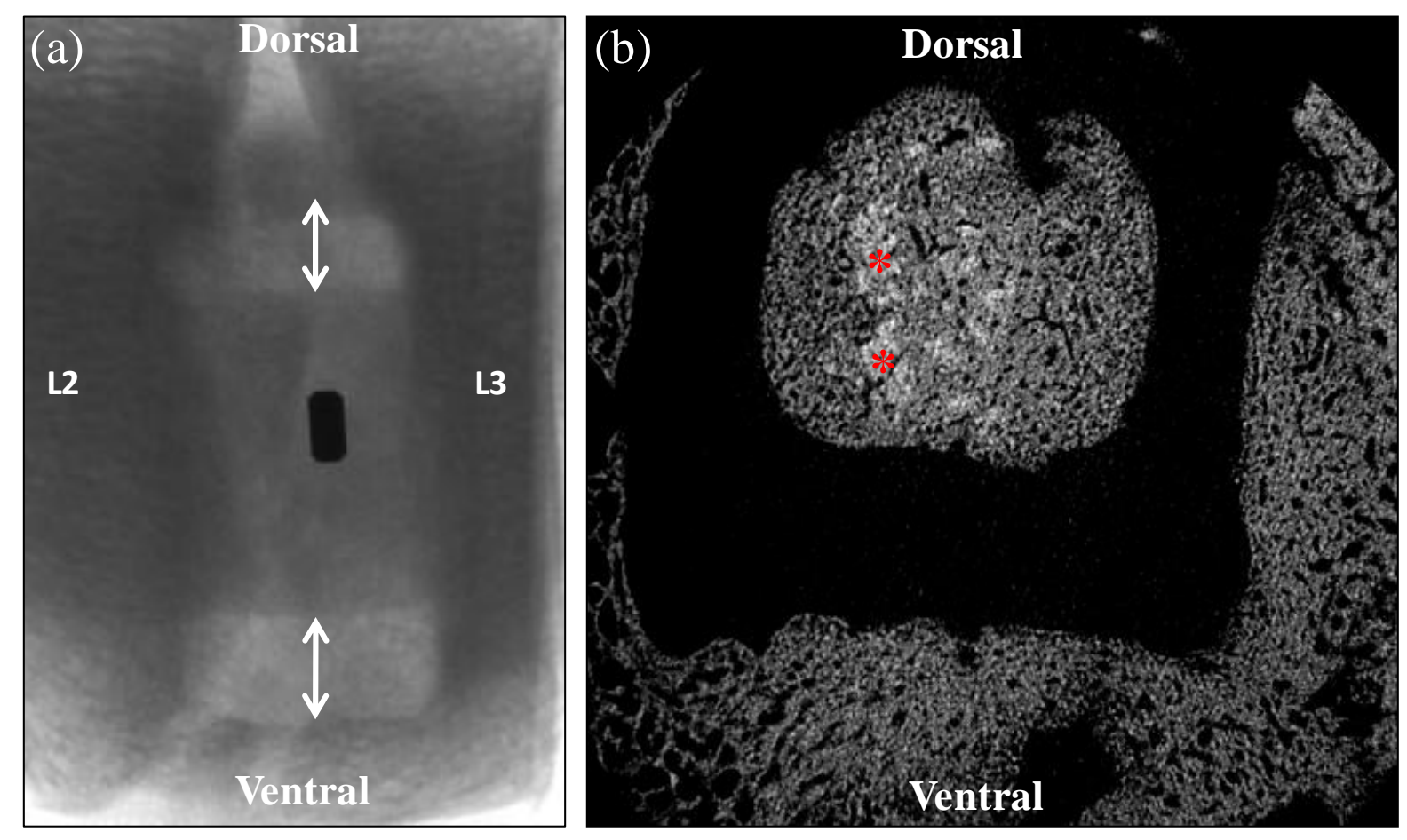

Figure 5. HE histological sagittal images of the different tested conditions (original magnification $\times 20$ ). The dorsal and ventral cage walls are visible $(*)$. Example of cages filled using (a) autologous bone graft which promoted bone fusion with an almost complete bone continuity between the two vertebral bodies showing a well-organized trabecular structure, 6 months after implantation; (b) autologous bone graft which led to non-union with fibrotic tissue surrounding the bone graft $(\rightarrow) 6$ months after implantation; (c) the blood-free CPC which did not promote any bone fusion although bone colonization developed at the periphery of the cage 3 months after implantation; (d) the CPC/blood composite which provided bone fusion with a new trabecular bone network bridging one vertebral body to the other, with only a few remaining cement particles at the ventral aspect of the cage $(\dagger), 3$ months after implantation. 

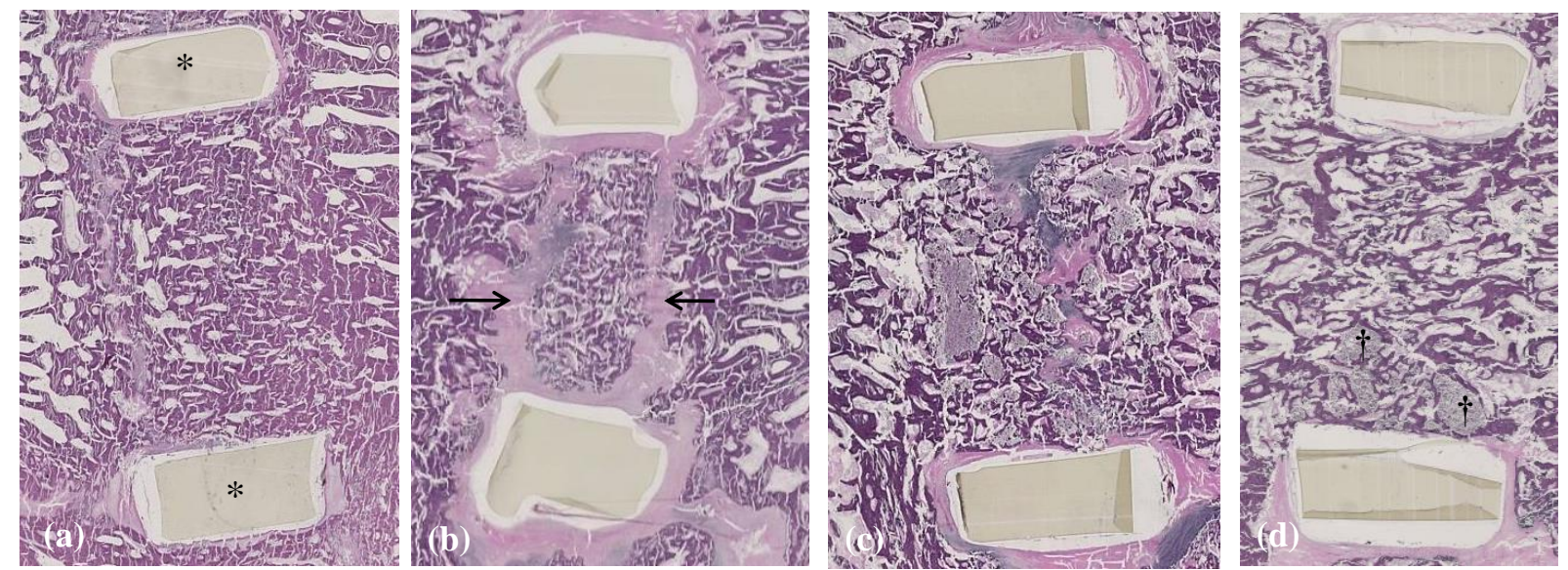

Figure 6. Movat'spentachrome stained histological sagittal images of the different tested conditions (original magnification $\times 20$ ). The dorsal and ventral cage walls are visible $(*)$. Mineralized bone tissue appears in light green, CPC are colored in blue-white. Example of cages filled using (a) autologous bone graft which promoted bone fusion with an obvious bone continuity between the two adjacent vertebral bodies 6 months after implantation; (b) the bloodfree CPC for which a high amount of residual CPC is visible ( $\dagger$ ) as well as a limited new bone formation mostly coming from the edges of the vertebral bodies without complete bone bridging, 3 months after implantation; (c) the CPC/blood composite with a new trabecular mineralized bone network bridging one vertebral body to the other, 3 months after implantation. 

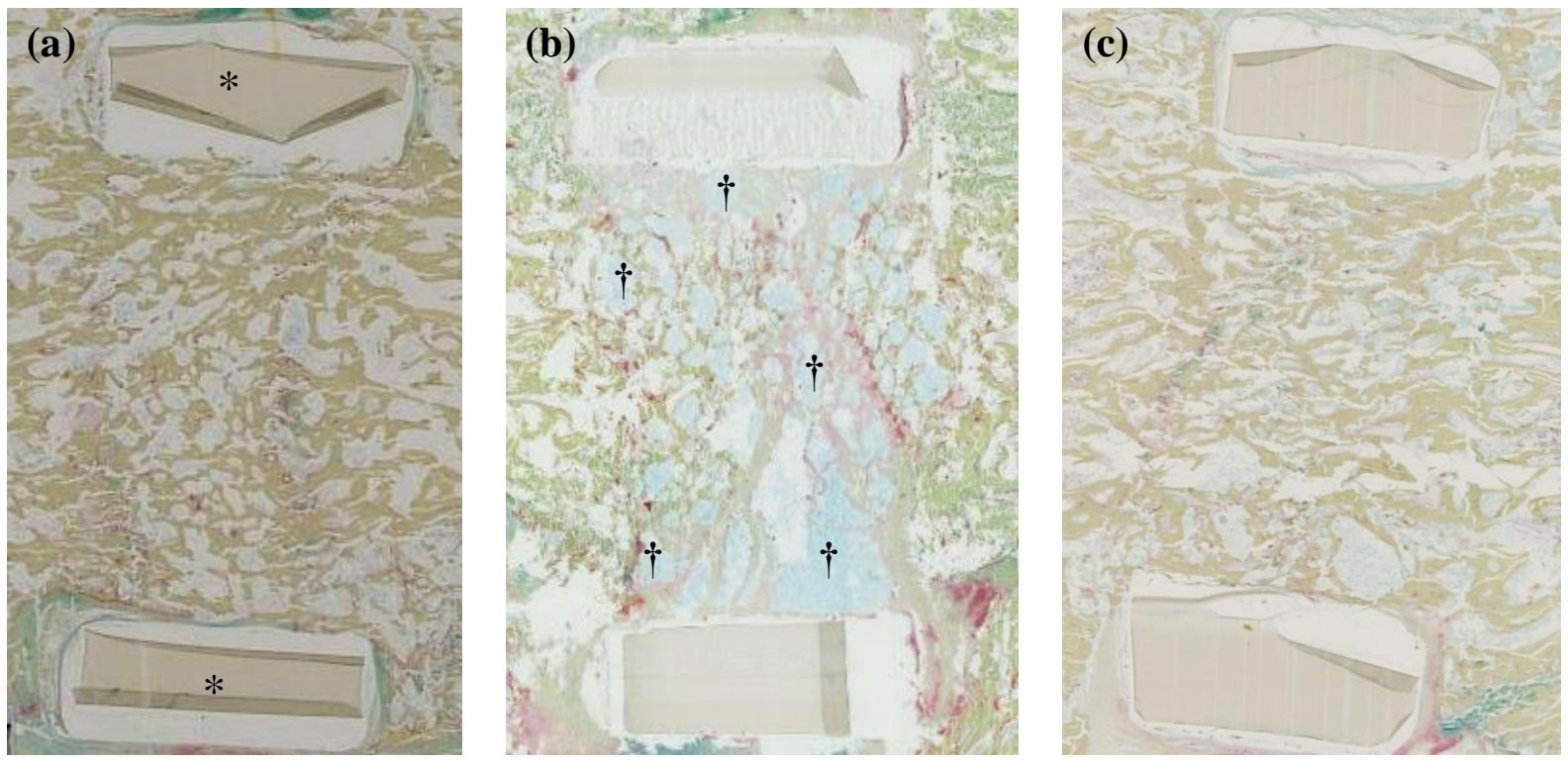

Figure 7. High magnification of a Movat's pentachrome stained histological image of new bone formation into a cage filled with the CPC-blood composite (see Figure 6c), 3 months after implantation. A few residual CPC fragments remain visible (†) but all of them are surrounded with well-mineralized newly-formed bone (in light green) in close contact with the cement surface. Osteoid borders $(\rightarrow)$ are visible on the newly-formed bone showing an active bone remodeling process. 


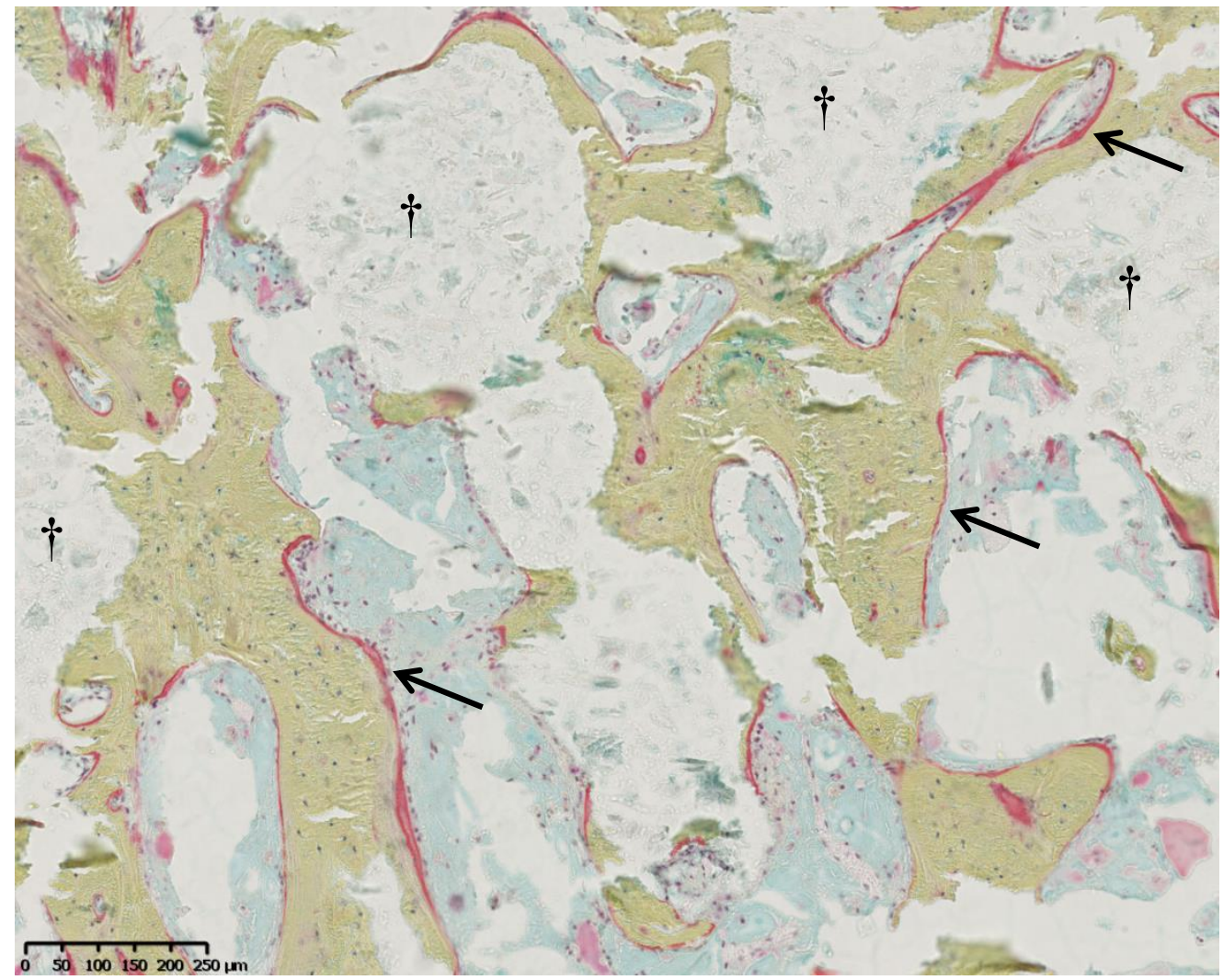

Table 1. Qualitative assessment (SEM analysis) of the bone fusion rate using cages filled with the three tested biomaterials: iliac bone autograft, $\mathrm{CPC} / \mathrm{blood}$ composite or $\mathrm{CPC}$ reference.

\begin{tabular}{|l|c|c|c|c|}
\hline Group (N=7) & \multicolumn{2}{|c|}{$\begin{array}{l}\text { CPC/blood composite versus CPC } \\
\text { reference, 3 months after surgery }\end{array}$} & \multicolumn{2}{|c|}{$\begin{array}{l}\text { CPC/blood composite versus bone } \\
\text { autograft, 6 months after surgery }\end{array}$} \\
\hline $\begin{array}{l}\text { CPC/blood } \\
\text { composite }\end{array}$ & CPC reference & $\begin{array}{l}\text { CPC/blood } \\
\text { composite }\end{array}$ & Bone autograft \\
\hline $\begin{array}{l}\text { Number of } \\
\text { successful }\end{array}$ & $6 / 7$ & $0 / 7$ & $5 / 7$ & $4 / 7$ \\
intervertebral & & & & \\
\hline
\end{tabular}




\section{Appendix A. Supplementary data}

\section{Materials and methods}

\section{Composition and preparation of the investigated bone substitutes}

The CPC reference was obtained from Graftys SA: Graftys ${ }^{\circledR}$ Quickset, a mixture of 78 wt.\% $\alpha-\mathrm{TCP}\left(\mathrm{Ca}_{3}\left(\mathrm{PO}_{4}\right)_{2}\right), 10$ wt.\% anhydrous dicalcium phosphate (DCPA) $\left(\mathrm{CaHPO}_{4}\right), 10$ wt.\% calcium-deficient hydroxyapatite (CDA) $\left(\mathrm{Ca}_{10-\mathrm{x}}[\mathrm{]}] \mathrm{x}\left(\mathrm{HPO}_{4}\right) \mathrm{y}\left(\mathrm{PO}_{4}\right)_{6-\mathrm{y}}(\mathrm{OH})_{2-\mathrm{z}}[\mathrm{]}\right.$ z), 2 wt.\% hydroxypropyl methyl cellulose (HPMC). The liquid phase consists of a 0.5 wt. $\% \mathrm{Na}_{2} \mathrm{HPO}_{4}$ aqueous solution (liquid/powder ratio $=0.45 \mathrm{~mL} \cdot \mathrm{g}^{-1}$ ). The cement paste was prepared by mixing $8 \mathrm{~g}$ of the powdered preparation with the liquid phase in a syringe for $2 \mathrm{~min}$, to ensure the homogeneity of the obtained paste before injection.

The CPC/blood composite investigated in this study is an experimental material composed of a mixture of 78 wt. $\% \alpha$ - TCP, 5 wt. \% dicalcium phosphate dihydrate (DCPD) $\left(\mathrm{CaHPO}_{4}, 2 \mathrm{H}_{2} \mathrm{O}\right)$, 5 wt.\% monocalcium monohydrate (MCPM) $\left(\mathrm{Ca}\left(\mathrm{H}_{2} \mathrm{PO}_{4}\right)_{2}, \mathrm{H}_{2} \mathrm{O}\right), 10$ wt.\% CDA, and 2 wt.\% HPMC. The liquid phase was autologous ovine whole blood stabilized by addition of sodium citrate (3.2 wt.\%) that was mixed in a syringe with the solid phase for 2 minutes. After waiting 25 minutes, the composite was ready for injection into the cage.

\section{Animal handling, surgical procedures and postoperative follow-up}

14 adult female Vendeen sheep with comparable size (average body weight of $60 \mathrm{~kg}$ ) were used in this study. Animals aged 7-11 years were used to get conditions close to humans, for which most of arthrodesis indications are related to spine degenerative phenomena occurring in middle-aged adults. Animal handling and surgical procedures were conducted at ONIRIS, 
according to the European Community Guidelines for the care and use of laboratory animals (2010/63/UE) and approved by the national ethical committee (Apafis 01336.01) and the animal welfare committee at the Oniris College of Veterinary Medicine. A 24 hours fasting period was applied before surgery to decrease the volume of the rumen content. Polyetheretherketone (PEEK) non-resorbable and radiolucent cages (ROI-C ${ }^{\circledR}$ from LDR Medical, Troyes, France) for human cervical arthrodesis by an anterior approach were selected for this study, since they had the appropriate dimensions for the developed animal model $(14 \times 14 \mathrm{~mm}, 5$ or $6 \mathrm{~mm}$ in height).

The surgery was carried out under general anesthesia, completed with intrathecal spinal anesthesia, with the animal in right lateral recumbency. A left paralumbar retroperitoneal approach was performed, since it allows easy access to vertebral bodies after retraction of the rumen, while preserving other abdominal organs. Each animal received two cages and the two implanted sites were separated by one preserved intervertebral disc (mostly $\mathrm{L}_{2}-\mathrm{L}_{3}$ and L4-L5) to limit the adverse effects of the surgery. When operating the lumbar levels, the corresponding spinal nerves were protected to expose the intervertebral disc. A classical discectomy was performed on both levels (figure 1a), before impaction of the cage by the lateral aspect of the rachis (figure 1c). For one-half of the animals, both intervertebral levels received an empty cage in which a hole had been drilled (figure 1c) preoperatively with a $1.5 \mathrm{~mm}$ diameter drill bit. This allowed injection of the CPC/blood composite in one level and the CPC reference in the other. Injection was performed using a syringe (figure 1d) fitted with a 18 gauge needle, and the injected volume was ca. $1.5 \mathrm{~mL}$ (cage volume $=0.5 \mathrm{~cm}^{3}$ ) to ensure complete filling of the space available between the two vertebrae. For the other seven animals, the CPC/blood composite was compared to an autologous bone graft, harvested from the ipsilateral iliac crest during the same operative session. Once harvested, the bone graft was morselized with a rongeur to fill the inner part of the cage before its impaction into the intervertebral space. No 
intraoperative complication was observed. The duration of the surgery was ca. 160 minutes and the average bleeding was less than $200 \mathrm{~mL}$.

A twice-daily clinical follow-up was carried for 20 days following surgery, to document changes in the animals gait and progression of wound healing. X-Ray examination of the operated lumbar segments was performed 15 days after surgery and before euthanasia to check the correct position of the cages into the operated intervertebral spaces. Explanted lumbar vertebral segments were collected and examined to detect possible residual mobility of operated and adjacent lumbar levels. The operated vertebral blocks, consisting of the intervertebral space and one centimeter of each adjacent vertebral body, were isolated using a bandsaw and placed in a $10 \%$ neutral formaldehyde solution before analysis of the specimen, including 3D microcomputed tomodensitometry ( $\mu$-CT), SEM observation and histological studies.

3. $\mu$-CT, Scanning Electron Microscopy and histological analyses

To minimize the possible effect of individual variation, two different implantation sites per animal were used, and each animal received two different biomaterials (CPC versus CPC/blood composite for the first group of animals, then CPC/blood composite versus autologous bone graft for the second group), with a randomization of the biomaterial allocation. A full-blind ex vivo analysis of the samples was performed by the same team for both groups.

\section{$3.1 \mu-C T$ analyses}

The vertebral blocks were placed in a pocket humidified with phosphate buffered saline, and the samples were imaged using X-ray radiation micro-CT (Skyscan 1272®, Bruker, Belgium). Scanning analysis was performed at $12-\mu \mathrm{m}$ isotropic resolution with the X-ray tube operated at $100 \mathrm{kV}$ and $100 \mu \mathrm{A}$. Image analysis was performed using the NRECON® and CTAn® softwares for image reconstruction and segmentation, and the CTVox ${ }^{\circledR}$ and DataViewer ${ }^{\circledR}$ softwares for $3 \mathrm{D}$ visualization of the scanned areas. Quantitative analysis of the bone content (bone volume 
$=\mathrm{BV}$ ) into the cage (total volume $=\mathrm{TV}$ ) was determined using both $2 \mathrm{D}$ and $3 \mathrm{D}$ measurements. Results were expressed as the percentage of new bone formation and percentage of remaining cement within the cages.

\subsection{Scanning Electron Microscopy analyses}

The fixed bone specimens were dehydrated with graded ethanol, and then infiltrated and embedded in glycol methyl-methacrylate (GMMA). The samples were cut in two pieces using a Leitz® diamond saw passing through the center of the intervertebral cage, along the sagittal axis. The resin blocks were polished and then sputtered with gold-palladium (Desk III ${ }^{\circledR}$ Denton vacuum, Moorestown, NJ, USA). The samples were observed by SEM (Leo 1450VP, Zeiss, Germany) and the images were acquired on the back-scattered electron mode. After an appropriate thresholding to differentiate the newly formed bone and the implant, quantitative SEM analysis from those images allowed to determine the respective amount of newly-formed bone and remaining cement into the considered cages.

A full image of the implant surface was obtained by reconstruction of ca. 700 pieces, using the Xn View ${ }^{\circledR}$ software. Analysis of the 2D microarchitecture was performed using the CTAn ${ }^{\circledR}$ software which allowed to differentiate the newly-formed bone from the remaining cement and to quantify their relative amount with a $2-\mu \mathrm{m}$ resolution. Reconstructed SEM images were used to define whether intervertebral bone fusion through the cage was effective for each one of the tested biomaterials, and determine the fusion rate for the three tested conditions.

\subsection{Histological analyses}

Undecalcified $5 \mu \mathrm{m}$ sections of each sample, were obtained using a hard tissue microtome (Leica Polycut SC2500) and were mounted on an adhesive tape to avoid stripping of the CPC. Sections were observed using hematoxylin-eosin (HE) staining and Movat'spentachrome staining which are perfectly adapted to distinguish bone mineral (yellow-green), osteoid tissue 
(red line) and cement (grey-blue). Sections were observed with a polarized light microscope (Axioplan2, Zeiss, Germany).

\subsection{Statistical analyses}

Quantitative assessment of the rate of new bone formation and cement resorption was performed by $\mu-\mathrm{CT}$ and SEM, using a non-parametric Wilcoxon signed-rank test for paired samples. A Pearson's correlation coefficient was calculated to measure the statistical relationship, between the new bone formation and the cement resorption within the cages. A p value less than 0.05 was considered significant. 
Figure S1. Quantitative $\mu$-CT measurements: correlation between the amount of new bone formation and cement degradation, into the inserted cages filled with either the blood-free $\mathrm{CPC}$ or the $\mathrm{CPC} / \mathrm{blood}$ composite.

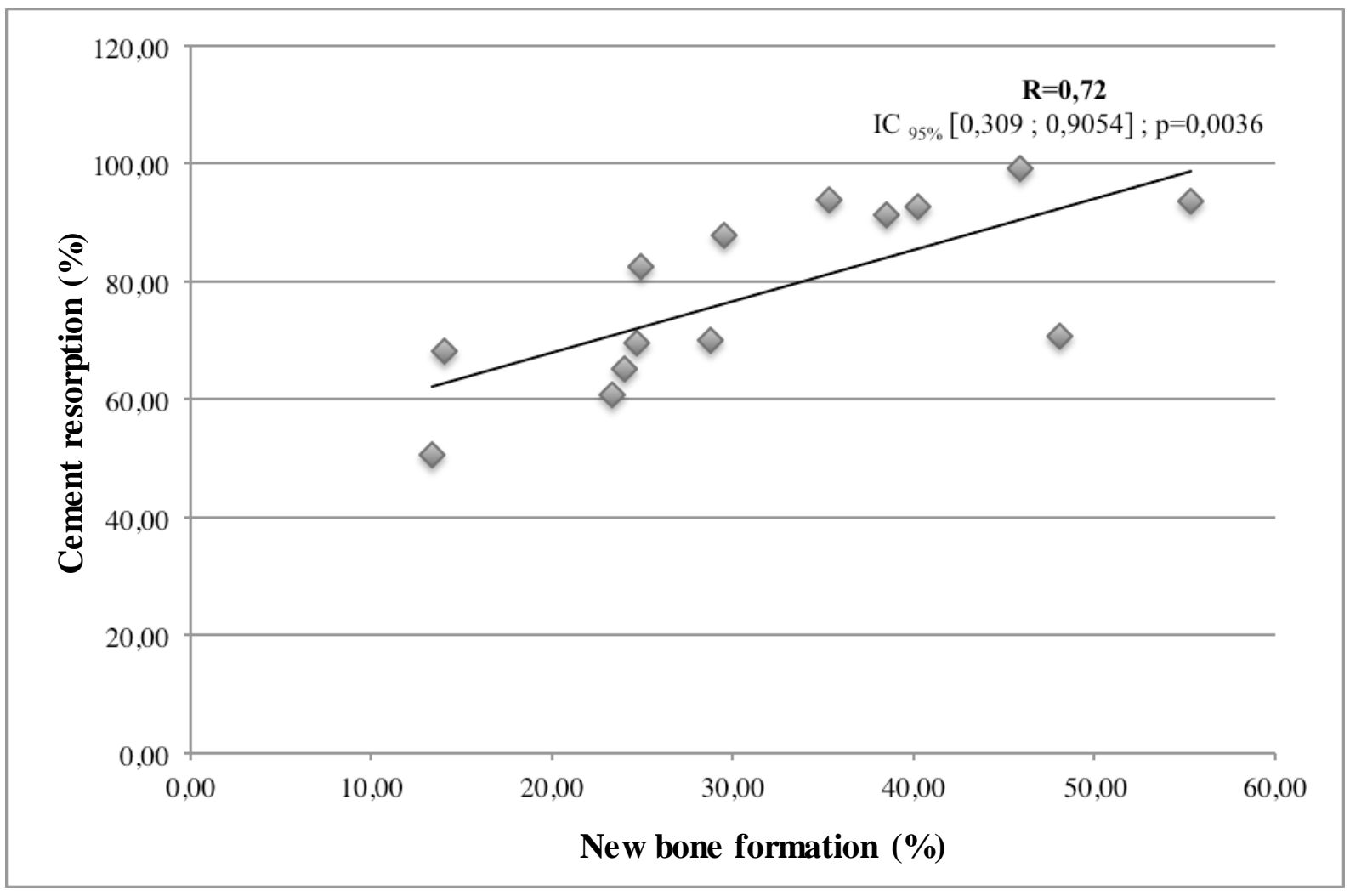

